\title{
Efficient $\mathrm{N}_{2}$ Formation on $\mathrm{Ag}(111)$ by Eley-Rideal
}

\section{Recombination of Hyperthermal Atoms}

\author{
María Blanco-Rey, ${ }^{*},+\ddagger$ Estibaliz Díaz, ${ }^{\ddagger}$ Gisela A. Bocan, ${ }^{\mathbb{I}}$ Ricardo Díez Muiño, ${ }^{\S, \ddagger}$ \\ Maite Alducin, ${ }^{\S, \ddagger}$ and J. Iñaki Juaristi $\AA^{\S, \dagger, \ddagger}$
}

Departamento de Física de Materiales, Facultad de Químicas UPV/EHU, Apartado 1072, 20018 Donostia-San Sebastián, Spain, Donostia International Physics Center (DIPC), Paseo Manuel de Lardizabal 4, 20018 Donostia-San Sebastián, Spain, CONICET and Centro Atómico Bariloche (CNEA), Av. Bustillo 9500, 8400 S.C. de Bariloche, Argentina, and Centro de Física de Materiales CFM/MPC (CSIC-UPV/EHU), Paseo Manuel de Lardizabal 5, 20018 Donostia-San Sebastián, Spain

E-mail: maria.blanco@ehu.es

\begin{abstract}
Using molecular dynamics simulations and potential energy surfaces of ab-initio quality, we show that direct pick-up of $\mathrm{N}$ adsorbates by gas-phase $\mathrm{N}$ is a highly efficient channel for $\mathrm{N}_{2}$ formation on $\operatorname{Ag}(111)$. This recombination process, called Eley-Rideal, was traditionally associated to lighter projectiles and regarded as marginal, but here we obtain reactivities for

\footnotetext{
${ }^{*}$ To whom correspondence should be addressed

${ }^{\dagger}$ Departamento de Física de Materiales, Facultad de Químicas UPV/EHU, Apartado 1072, 20018 Donostia-San Sebastián, Spain

‡ Donostia International Physics Center (DIPC), Paseo Manuel de Lardizabal 4, 20018 Donostia-San Sebastián, Spain

Il CONICET and Centro Atómico Bariloche (CNEA), Av. Bustillo 9500, 8400 S.C. de Bariloche, Argentina

$\S$ Centro de Física de Materiales CFM/MPC (CSIC-UPV/EHU), Paseo Manuel de Lardizabal 5, 20018 DonostiaSan Sebastián, Spain
} 
$\mathrm{N}$ of $\gtrsim 35 \%$ even at incident energies of a few $\mathrm{eV}$. The good agreement found between simulated and published experimental energies of the desorbed $\mathrm{N}_{2}$ is a fingerprint of this otherwise elusive recombination.

\section{TOC Graphic}

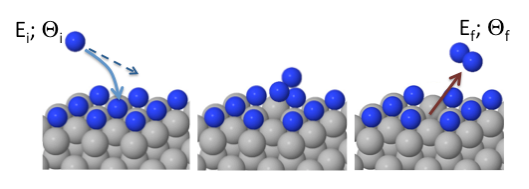

Keywords: Gas-surface interactions, Eley-Rideal reactions, scattering, potential energy surfaces, molecular dynamics. 
Among the various elementary processes that occur in everyday gas-surface reactions, those resulting in the recombination and desorption of molecules deserve a special attention, as they regulate the replenishment of the surface active sites. One of these processes, the direct Eley-Rideal (ER) recombination has been found to be a marginal phenomenon, only observed experimentally for incoming light atoms (H,D). ${ }^{1-9}$ Originally postulated as an atom exchange, ${ }^{10-12}$ the ER process is nowadays assigned to the direct recombination of an incoming gas-phase species that picks up a surface adsorbate to conform a molecular compound that desorbs during the collision. Even if the initial measurements pointed to ER cross sections of about $2-5 \AA^{2},{ }^{4,6,9}$ subsequent theoretical studies demonstrated that they were around one order of magnitude smaller because most of the recombination events proceed after few collisions of the incoming (H,D) atom with the surface, i.e., through the slower hot-atom (HA) process. ${ }^{13-18}$

In the scope of reactive ion scattering (RIS), $\mathrm{C}$ abstraction from a graphite surface by $\mathrm{N}^{+}$ ions has been characterised by experiments, and found to happen after several collisions with the substrate $^{19}$ Recombination of neutral atomic species heavier than (H,D) has been experimentally studied in the context of $\mathrm{CO}$ formation and $\mathrm{CO}$ oxidation on metal surfaces. In all cases, $\mathrm{CO}$ and $\mathrm{CO}_{2}$ formation is dominated by the Langmuir-Hinshelwood (LH) recombination in which the species involved in the reaction are fully thermalized with the surface. ${ }^{20-22}$ Also in these systems, the direct ER pick-up events are accepted to play a very minor role. Within this context, the recent experiments by Ueta et al. suggesting the existence of direct recombination on the N-covered $\operatorname{Ag}(111)$ surface between gas-phase and adsorbed $\mathrm{N}$ atoms are certainly intriguing. ${ }^{23,24}$ All the more so when recent simulations of $\mathrm{N}_{2}$ formation on the $\mathrm{W}(100)$ surface also exhibit low ER probabilities. $^{25}$

In this letter we demonstrate that thermal and hyperthermal gas-phase $\mathrm{N}$ scattered off $\mathrm{N}$ covered $\mathrm{Ag}(111)$ is indeed a prototype system that fulfills all the requirements to observe a highly efficient direct ER recombination. This outcome poses a new scenario for the study of gas-surface interactions, as it shows that fast direct recombination channels may be active in contrast to the usual assumption of neglecting them, specially for large atomic projectiles. 
The complex modeling of the ER mechanism has been successfully attained by a careful mixing of the ab-initio potential energy surfaces calculated for gas-phase $\mathrm{N}$ and $\mathrm{N}_{2}$ impinging, respectively, on the $(1 \times 1) \mathrm{N}$-covered and clean $\operatorname{Ag}(111)$ surfaces. Importantly, our calculations nicely reproduce the recent measurements by Ueta et al. using $\mathrm{N}$ and $\mathrm{N}_{2}$ mixed molecular beams reflected off clean and $\mathrm{N}$-covered $\operatorname{Ag}(111) .{ }^{23,24}$ In these experiments a small amount of molecules is detected at outgoing polar angles $\Theta_{f}<40^{\circ}$ off $\mathrm{N}$-covered $\operatorname{Ag}(111)$, but not off clean $\operatorname{Ag}(111)$ (see filled and open triangles in Figure 1). The authors cautiously interpreted the existence of those molecules as an indirect evidence of a recombination process taking place on the N-covered surface. The calculations in the present letter prove unambiguously that the origin of those molecules is a surface reaction. Quantitative agreement with the experimental energy angular spectra supports the interpretation. Moreover, we show that at least $1 / 3$ of the impinging atoms result in an ER event. All in all, the present molecular dynamics (MD) study confirms that Ref. ${ }^{24}$ contains the first experimental fingerprint of $\mathrm{N}_{2}$ formed by ER reaction, a process that up to now has been exclusively detected for incoming light atoms.

First, we have simulated the scattering dynamics of $\mathrm{N}_{2}$ molecules off clean $\operatorname{Ag}(111)$. The interaction between $\mathrm{N}_{2}$ and $\mathrm{Ag}(111)$ has been modeled by a six-dimensional (6D) potential energy surface (PES), which has been constructed from interpolation of ab-initio energies evaluated for a dense set of $\{x, y, z\}$ coordinates of the $\mathrm{N}$-atoms and frozen Ag positions. Those $a b$-initio energies have been calculated within the density functional theory using ultrasoft pseudopotentials ${ }^{26}$ and the PW91 generalized gradient approximation ${ }^{27}$ for the exchange and correlation functional. The interpolation has been carried out using the corrugation reduction procedure method. ${ }^{28} \mathrm{We}$ find that the molecule-surface interaction is, overall, strongly repulsive. Further details on the calculations can be found in Ref. ${ }^{29}$

Classical 6D MD simulations have been performed with molecular projectiles that are set to match the experimental settings, including incoming polar angles $\Theta_{i}=60^{\circ}$ and a broad distribution of translational kinetic energies with average value $\left\langle E_{i}\right\rangle=5.6 \mathrm{eV}$ and $f w h m=6.3 \mathrm{eV}$, i.e. we use an effusive beam. ${ }^{24}$ Initially, the $\mathrm{N}_{2}$ molecules are not rotationally or vibrationally excited. 
An optimal description of the dynamics is achieved when including an energy loss channel into Ag lattice vibrations by means of the Generalized Langevin Oscillator (GLO) model ${ }^{30-33}$ using the same implementation of Refs., ${ }^{34-36}$ where the surface vibration frequencies are taken from experimental values for $\operatorname{Ag}(111) .{ }^{37}$ The agreement between theory and experiment is exceedingly good for the resulting angular distribution of final-to-initial translational energy ratios, $\left\langle E_{f}\right\rangle /\left\langle E_{i}\right\rangle$, as shown in Figure 1. The analysis of the angular distribution of scattered molecules together with additional calculations for monoenergetic beams explain the distinctive $V$-shaped experimental distributions, nicely reproduced here by theory. Since the molecules in the beam with higher $E_{i}$ can access more corrugated regions of the PES, they produce broader angular distributions than the less energetic ones, as shown in the middle panel of Figure 1. For this reason, on average, the effusive molecular beam has a $\lesssim 40^{\circ}$ spread and, importantly, the tails of the distribution are dominated by the more energetic molecules. As a result the final-to-initial translational energy ratio increases the more the scattered $\mathrm{N}_{2}$ departures from specularity. In agreement with the experiment, there is no hint of dissociative sticking in the simulations and no molecules are detected at $\Theta_{f}<40^{\circ}$.

On the $\mathrm{N}$-covered surface, however, a small amount of $\mathrm{N}_{2}$ molecules leave the $\mathrm{N}$-covered surface at $\Theta_{f}<40^{\circ}$ with intensities about 50 times smaller than the specular peak. ${ }^{24}$ The offspecular signal is consistent with a corrugated PES, as it is the case for $\mathrm{N}$-atoms but not for $\mathrm{N}_{2}$ molecules, which suggests an underlying molecule formation on the surface. In this respect, MD studies on the $(1 \times 1) \mathrm{N}$-covered $\operatorname{Ag}(111)$ surface with a 3D PES have shown that every adsorption trajectory ends up in a $\mathrm{N}(\mathrm{g})-\mathrm{N}(\mathrm{a})$ bond, where $\mathrm{N}(\mathrm{g})$ and $\mathrm{N}(\mathrm{a})$ denote gas-phase $\mathrm{N}$ atoms and $\mathrm{N}$ adsorbates, respectively. Despite the limitations of a 3D description, which keeps N(a) at frozen coordinates, this theoretical result also points to $\mathrm{N}_{2}$ formation. ${ }^{36}$ The progressive cleaning of the $\mathrm{N}$ (a) via $\mathrm{N}_{2}$ formation also explains the experimental observation that the $\mathrm{N}$-atom scattering data on $\mathrm{N}$-covered $\operatorname{Ag}(111)$ resemble that of the clean surface. Those recombination processes compete in turn with the replenishment of clean $\mathrm{Ag}(111)$ patches by $\mathrm{N}(\mathrm{g})$. Thus, a fundamental question that raises here is the efficiency of the recombination processes. We show next that the ER mechanism, which sets a lower boundary value of the total $\mathrm{N}_{2}$ yield, is unusually efficient. 
A good formulation of the problem should contain both the large potential corrugation brought by the $\mathrm{N}$-coverage and $\mathrm{N}$ (a) mobility to account for eventual desorption events. With this in mind, our model PES uses energies of ab-initio quality and is constructed as sketched in Figure 2 by merging (i) the 3D PES for $\mathrm{N}(\mathrm{g})$ approaching the $(1 \times 1)$ N-covered $\mathrm{Ag}(111)$ for $\mathrm{N}(\mathrm{g})-\mathrm{N}(\mathrm{a})$ distances $d>R_{s}$ and (ii) the 6D PES for the $\mathrm{N}_{2}$ molecule approaching the clean surface for $d<R_{s}$. When $\mathrm{N}(\mathrm{g})$ crosses the boundary from the 3D to the $6 \mathrm{D}$ region, $\mathrm{N}(\mathrm{a})$ is allowed to move and may become an atom of a $\mathrm{N}_{2}$ molecule. The choice $R_{s}=2.2 \AA$ for the boundary ensures negligible discontinuities when integrating the equations of motion. Details on the PES continuity and the settings of the MD simulations performed on the merged PES can be found in the SI. ${ }^{29}$

Figure 3 shows the fraction of atomic trajectories that result in $\mathrm{N}_{2}$ formation by ER, where we observe that $\gtrsim 35 \%$ of the projectiles in the experimental effusive beam used in the calculation succeed to react with surface species. It is a fast process with typical simulation times below $<0.4 \mathrm{ps}$ for the effusive atomic beam, and therefore the rigid surface approximation is well justified. Inclusion of GLO in the dynamics has a non-negligible impact, albeit small, in the reaction probability for $E_{i}<2 \mathrm{eV}$. For those $E_{i}$ values, energy exchange with the $\mathrm{Ag}(111)$ lattice reduces the ER efficiency by $\sim 10 \%$. This uncommonly large efficiency, specially at incident energies as high as $E_{i}=7 \mathrm{eV}$, is a non-trivial outcome of the dynamics of the system. Slower $\mathrm{N}(\mathrm{g})$ with $E_{i}<2 \mathrm{eV}$ increase further the reaction probability. Low-energy projectiles visit lower energy regions of the PES, so that the dynamics is dictated by the highly exothermic and barrierless character of the reaction $\left(9.8 \mathrm{eV}\right.$ are dissipated by a single $\mathrm{N}_{2}$ formation, whilst only just $2.01 \mathrm{eV}$ are needed to desorb each $\left.\mathrm{N}(\mathrm{a})^{38}\right)$. On similar grounds, $\mathrm{CO}$ formation by ER at C-covered $\mathrm{Pt}(111)$ with $E_{i}(\mathrm{O})<0.5 \mathrm{eV}$ was predicted to proceed with $97 \%$ efficiency by early MD simulations. ${ }^{39}$ However, later molecular beam experiments showed that $\mathrm{LH}$ was the dominant mechanism for $\mathrm{CO}$ recombination in this system. ${ }^{20}$ On N-covered $\mathrm{W}(100)$, the existence of an energy barrier makes $\mathrm{N}_{2}$ formation by ER less efficient than on $\mathrm{N}$-covered $\mathrm{Ag}(111)$, despite the reaction being also exothermic. ${ }^{25,40}$ In the case of $\mathrm{H}_{2} / \mathrm{Cu}(111)$, the $\mathrm{H}-\mathrm{H}$ interaction is repulsive when the atoms lie close to the metal, and most recombinations are driven by HA. ${ }^{14}$ In other words, the efficiency of the reaction de- 
pends on the balance between several interactions: atom-surface, atom-atom, etc. The nature of the recombination mechanism relies thus in the chemical interaction between the neutral atoms on the surface. Effective ER with heavy ions has been measured in RIS. However, the ER scenario for ions in RIS is different to the one with neutral atoms, since the recombination mechanism is due to the electrostatic ion-adsorbate attraction (ion-dipole) and it is effective only for physisorbed atoms. $^{41,42}$

The angular dependence of the molecular translational energy is accounted for in the molecular beam experiments by Ueta et al., ${ }^{24}$ and it is in reasonable agreement with the theoretical value from MD simulations that use the atomic effusive incident beam, as shown in Figure 1 for $\Theta_{f}<40^{\circ}$ inplane directions. At $\Theta_{f}>40^{\circ} \mathrm{N}_{2}$ molecules originated by ER contribute only to $\lesssim 3 \%$ of the total $\mathrm{N}_{2}$ intensity, and therefore those $\left\langle E_{f}\right\rangle /\left\langle E_{i}\right\rangle$ values are dominated by $\mathrm{N}_{2}$ reflection. This is due to the fact that most formed $\mathrm{N}_{2}$ molecules leave the surface at out-of-plane directions, and thus they are not to be observed in the experimental spectra. The angular distribution of inplane $\mathrm{N}_{2}$ molecules formed by ER is shown at the top panel of Figure 1. These intensities are about two orders of magnitude smaller than the intensities of in-plane scattered $\mathrm{N}_{2}$ (middle panel), in reasonable agreement with the experimental observations. ${ }^{24}$ The theoretical $\left\langle E_{f}\right\rangle /\left\langle E_{i}\right\rangle$ values at $\Theta_{f}<40^{\circ}$ are somewhat larger than the experimental ones because secondary recombination processes are not captured by the current PES model. These would yield, if any, slower products as the HA longer surface residence times allow for further energy dissipation into the substrate. The good agreement in $\left\langle E_{f}\right\rangle /\left\langle E_{i}\right\rangle$ strongly indicates that most of those observed $\mathrm{N}_{2}$ molecules at $\Theta_{f}<40^{\circ}$ result from a ER reaction.

Reactive trajectory tracking unveils the ER mechanism at the atomic scale. For a N(g)-N(a) pair, a ER reaction impact parameter, $b$, is defined as sketched in Figure 2. For a given target atom N(a), Figure 4 shows the initial impact parameter distributions of all the incident atoms, $P_{0}(b)$, and the reactive ones (i.e. those that will result in an ER event), $P_{r}(b)$. The potential outside the shaded region in Figure 2 is able to steer the $\mathrm{N}(\mathrm{g})$ projectile, deflecting it towards a target other than the one it was initially set to collide with. If the potential far from the surface were negligible, the $\mathrm{N}(\mathrm{g})$ 
atoms would move along straight trajectories and would impinge on N(a) with impact parameters $R_{t h} \leq b \leq R_{s}$ due to the shadowing effect of neighbouring adsorbates. The steering effect comes through as an $E_{i}$-dependent shift towards larger $b$ values in the distributions, so that they start at $b>R_{t h}$ and take values $b>R_{s}$. The lower the $E_{i}$, the stronger the steering, which evidences the long range attraction exerted by a dense $\mathrm{N}(\mathrm{a})$ coverage. The distribution $P_{0}(b)$ is maximal at $b \simeq 2 \AA$, under the current incidence geometry. $P_{r}(b)$ is double peaked, and the peak position and width changes with $E_{i}$. The peaks in $P_{r}(b)$ can be assigned to two distinct recombination mechanisms. At large $b$ values, the $\mathrm{N}(\mathrm{g})$ momentum component along the $\mathrm{N}(\mathrm{g})-\mathrm{N}(\mathrm{a})$ direction is small, and therefore the net attractive force between both atoms acts long enough as to produce the adsorbate abstraction. At low $b$ values, $\mathrm{N}(\mathrm{g})$ approaches $\mathrm{N}(\mathrm{a})$ fast enough to enter the repulsive region of the PES. A collision occurs that destabilizes N(a) from its equilibrium adsorption site (it retracts downwards by an average $\Delta z=0.2$ and $0.4 \AA$ for $E_{i}=1$ and $4 \mathrm{eV}$, respectively) and the recoiling $\mathrm{N}(\mathrm{g})$ can easily drag it along. This peak height is smaller at larger $\mathrm{E}_{i}$, since the recoil may be too fast for $\mathrm{N}(\mathrm{a})$ to follow the projectile. If we consider the quotient $P_{r}(b) / P_{0}(b)$ between the distributions of the reactive and all the incoming $\mathrm{N}(\mathrm{g})$-atoms, it turns out that the collision (abstraction) mechanism has an approximate maximum efficiency of $75 \%(100 \%)$ at all $E_{i}$ values. Nevertheless, the global likelihoods of these two mechanisms in the actual dynamics are very different, as only a few $\mathrm{N}(\mathrm{g})$ atoms are beamed onto the surface at small $b$ initial conditions [see $P_{0}(b)$ curves in Figure 4]. Double peaked distributions have been reported in the formation of $\mathrm{HCl}$ on $\mathrm{Au}(111)$ by $\mathrm{ER},{ }^{15}$ too. In contrast, a small $b$ mechanism is not possible at $\mathrm{W}(100)$, since repulsion between $\mathrm{N}$-atoms exists at vertical distances $2.0-2.5 \AA$ that deflects $\mathrm{N}(\mathrm{g})$ towards the $\mathrm{N}(\mathrm{a})$ sides. $^{25}$

The decrease in efficiency when energy exchange with the lattice is included in the model using GLO (see Figure 3) is mainly associated to the collision reactive channel. As shown in Figure 4 the low- $b$ peak height is decreased upon inclusion of GLO, while the high- $b$ peak of $P_{r}(b)$ remains unaffected. Since the lattice is allowed to move, a fraction of the energy released in the collision is transferred to the substrate and N(a) does not acquire enough energy to become unbound from the 
hollow site. The N-N bond formation on $\operatorname{Ag}(111)$ is exothermic by $\sim 7 \mathrm{eV}$, thus large amounts of energy are released that are shared between the product and the substrate. On the one hand, we have checked that the formed $\mathrm{N}_{2}$ products leave the molecule at high rovibrationally excited states. On the other hand, we can extract information on the average energy transferred to the substrate upon molecule formation by comparing the formed $\mathrm{N}_{2}$ average kinetic energies in GLO and adiabatic frozen surface simulations. We find that this energy transfer is $1.57 \mathrm{eV}$ for an incident effusive atomic beam of $\left\langle E_{i}\right\rangle=4.3 \mathrm{eV}$. For a monoergetic beam of $E_{i}=7.0 \mathrm{eV}, 2.42 \mathrm{eV}$ can be transferred to substrate lattice vibrations. This energy is large enough to induce desorption of $\mathrm{N}$ adsorbates and create hot atoms, increasing $\mathrm{N}_{2}$ production by indirect mechanisms that are beyond the scope of the present work.

Since it is difficult to distinguish experimentally HA from ER products, ${ }^{43,44}$ MD studies come in as a valuable tool in the study of gas-surface reaction pathways. They have shown that the ER cross-section for $\mathrm{HCl}$ formation on $\mathrm{Au}(111)$ is $\sigma<1 \AA^{2}$ (about $10 \%$ of the $\mathrm{HCl}$ yield), ${ }^{16}$ instead of the initially reported experimental value $\sigma=2 \AA^{2} .{ }^{4}$ For $(H, D)$ reacting on $\mathrm{Cu}(111)$, theory also yields smaller cross-sections ( $\sigma<0.5 \AA^{2}$, equivalent to $3.2-4.4 \%$ of the $\mathrm{H}_{2}$ or HD products) ${ }^{13,14}$ than experiments $\sigma=5 \AA^{2} .^{2,5}$ Less than $5 \%$ of the $\mathrm{N}_{2}$ formed on W(100) follows from ER with cross-sections $\sigma=0.4 \AA^{2}{ }^{25}$ Considering this background, it is remarkable to find that the single collision ER route towards $\mathrm{N}_{2}$ on $\operatorname{Ag}(111)$ with efficiency $>35 \%\left(\sigma>2.5 \AA^{2}\right)$ as shown in Figure 3, even with the use of much more energetic projectiles than in the aforementioned reactions. A competingly efficient $\mathrm{N}$-atom sticking process on clean $\mathrm{Ag}(111)$ is present for $E_{i} \leq 1.5 \mathrm{eV}$, but drops fast to negligible values for $E_{i} \geq 2 \mathrm{eV} .{ }^{38}$ The ER efficiency obtained in this work is a lower boundary value to the total $\mathrm{N}_{2}$ yield, that may also result from $\mathrm{HA}$ or $\mathrm{LH}$. Therefore, the surface cleaning may be significant also for projectiles below $2 \mathrm{eV}$ and a fast adsorbate removal from the $\mathrm{N}$-covered $\operatorname{Ag}(111)$ is foreseen that would explain the experimental observation that reflected $\mathrm{N}$ atom distributions on clean and $\mathrm{N}$-covered $\mathrm{Ag}(111)$ are almost identical ${ }^{24}$ despite the significant differences in their PES topographies. ${ }^{36}$ An accurate description of the cleaning-replenishment cycles would require knowledge of the kinetic constants of the individual events. Nevertheless, since 
$\mathrm{N}_{2}$ recombination probabilities are larger than $\mathrm{N}$ sticking probabilities on $\operatorname{Ag}(111),{ }^{38}$ it seems plausible that cleaning dominates as the experiment is being conducted on the covered surface.

In summary, we have shown that impinging $\mathrm{N}$ projectiles of $\sim \mathrm{eV}$ energies on $\mathrm{N}$-covered $\operatorname{Ag}(111)$ can result in the efficient formation of $\mathrm{N}_{2}$ by fast pick-up events, also known as EleyRideal (ER) processes. We find that at least $35 \%$ of the incident $\mathrm{N}$ will recombine following this mechanism. This result sharply contrasts with what is known for other gas-surface systems where ER has a lesser weight and is typically restricted to light projectiles. The high $\mathrm{N}_{2}$ yield found here is mainly due to the relatively long-ranged attractive and barrierless nature of the N-N interaction potential in the presence of the $\operatorname{Ag}(111)$ surface. This finding establishes the necessary conditions for efficient ER recombination and, importantly, poses a new scenario for many surface reactions, where now fast direct pick-up should be seen as a relevant product desorption channel.

\section{Acknowledgement}

L. Martin-Gondre, P. Larrégaray and A.W. Kleyn are kindly acknowledged for stimulating discussions. Work supported in part by the Basque Departamento de Educación, Universidades e Investigación, the University of the Basque Country UPV/EHU (Grant No. IT-366-07) and the Spanish Ministerio de Ciencia e Innovación (Grant No. FIS2010-19609-C02-02). M.B.-R. acknowledges the Gipuzkoako Foru Aldundia and the European Commission (Grant No. FP7-PEOPLE-2010RG276921). G.A.B. acknowledges ANPCYT (Grant No. PICT 2010-1558). Computational resources were provided by the DIPC computing center.

\section{Supporting Information Available}

The Supporting Information contains: (i) the settings used in the construction of the 6D PES of a $\mathrm{N}_{2}$ molecule that interacts with the $\operatorname{Ag}(111)$ surface and (ii) a test on the trajectory continuity at the boundary $R_{s}$ between the 3D and 6D PESs (see Figure 2). This material is available free of charge via the Internet at http://pubs . acs.org. 


\section{References}

(1) Lykke, K.; Kay, B. D. State-To-State Inelastic and Reactive Molecular Beam Scattering from Surfaces. SPIE Proc. 1990, 1208, 18.

(2) Rettner, C. T. Dynamics of the Direct Reaction of Hydrogen Atoms Adsorbed on $\mathrm{Cu}(111)$ with Hydrogen Atoms Incident from the Gas-Phase. Phys. Rev. Lett. 1992, 69, 383-386.

(3) Rettner, C. T.; Auerbach, D. J. Distinguishing the Direct and Indirect Products of a GasSurface Reaction. Science 1994, 263, 365-367.

(4) Rettner, C. T. Reaction of an H-atom Beam with Cl/Au(111) - Dynamics of Concurrent EleyRideal and Langmuir-Hinshelwood Mechanisms. J. Chem. Phys. 1994, 101, 1529-1546.

(5) Rettner, C. T.; Auerbach, D. J. Dynamics of the Eley-Rideal Reaction of D-atoms with Hatoms Adsorbed on $\mathrm{Cu}(111)$ - Vibrational and Rotational State Distributions of the HD Product. Phys. Rev. Lett. 1995, 74, 4551-4554.

(6) Rettner, C. T.; Auerbach, D. J. Dynamics of the Formation of HD from D(H) Atoms Colliding with $\mathrm{H}(\mathrm{D}) / \mathrm{Cu}(111)$ : A Model Study of an Eley-Rideal Reaction. Surf. Sci. 1996, 357, 602608.

(7) Rettner, C. T.; Auerbach, D. J. Quantum-State Distributions for the HD Product of the Direct Reaction of $\mathrm{H}(\mathrm{D}) / \mathrm{Cu}(111)$ with $\mathrm{D}(\mathrm{H})$ Incident from the Gas Phase. J. Chem. Phys. 1996, 104, 2732-2739.

(8) Kammler, T.; Lee, J.; Küppers, J. A Kinetic Study of the Interaction of Gaseous H(D) Atoms with D(H) Adsorbed on Ni(100) Surfaces. J. Chem. Phys. 1997, 106, 7362-7371.

(9) Kammler, T.; Küppers, J. Interaction of H Atoms with $\mathrm{Cu}(111)$ Surfaces: Adsorption, Absorption, and Abstraction. J. Chem. Phys. 1999, 111, 8115-8123.

(10) Eley, D. D.; Rideal, E. K. Parahydrogen Conversion on Tungsten. Nature 1940, 146, 401-402. 
(11) Eley, D. D.; Rideal, E. K. The Catalysis of the Parahydrogen Conversion by Tungsten. Proc. R. Soc. London, Ser. A 1941, 178, 429-451.

(12) Eley, D. D. The Interchange of Hydrogen in the Adsorbed Film on Tungsten. Proc. R. Soc. London, Ser. A 1941, 178, 452-464.

(13) Persson, M.; Jackson, B. Isotope Effects in the Eley-Rideal Dynamics of the Recombinative Desorption of Hydrogen on a Metal Surface. Chem. Phys. Lett. 1995, 237, 468-473.

(14) Shalashilin, D. V.; Jackson, B.; Persson, M. Eley-Rideal and Hot-Atom Reactions of H(D) Atoms with D(H)-Covered Cu(111) Surfaces; Quasiclassical Studies. J. Chem. Phys. 1999, $110,11038-11046$.

(15) Lemoine, D.; Quattrucci, J. G.; Jackson, B. Efficient Eley-Rideal Reactions of H Atoms with Single Cl Adsorbates on Au(111). Phys. Rev. Lett. 2002, 89, 268302.

(16) Quattrucci, G.; Jackson, B. Quasiclassical Study of Eley-Rideal and Hot Atom Reactions of H Atoms with Cl Adsorbed on a Au(111) Surface. J. Chem. Phys. 2005, 122, 074705.

(17) Guvenc, Z. B.; Sha, X.; Jackson, B. Eley-Rideal and Hot Atom Reactions between Hydrogen Atoms on Ni(100): Electronic Structure and Quasiclassical Studies. J. Chem. Phys. 2001, $115,9018-9027$.

(18) Martinazzo, R.; Assoni, S.; Marinoni, G.; Tantardini, G. F. Hot-Atom versus Eley-Rideal Dynamics in Hydrogen Recombination on Ni(100). I. The Single-Adsorbate Case. J. Chem. Phys. 2004, 120, 8761-8771.

(19) Z.-W. Deng,; Souda, R. Eley-Rideal Abstraction of Carbon from Graphite by Hyperthermal $\mathrm{N}^{+}$ions. J. Chem. Phys 2002, 117, 6235-6238.

(20) Kori, M.; Halpern, B. L. Vibrational Energy Distribution of CO in the Oxidation of C on Pt. Chem. Phys. Lett. 1983, 98, 32-36. 
(21) Stampfl, C.; Scheffler, M. Anomalous Behavior of Ru for Catalytic Oxidation: A Theoretical Study of the Catalytic Reaction $\mathrm{CO}+1 / 2 \mathrm{O}(2) \rightarrow \mathrm{CO}_{2}$. Phys. Rev. Lett. 1997, 78, 1500-1503.

(22) Wheeler, M. C.; Reeves, C. T.; Seets, D. C.; Mullins, C. B. Experimental Study of CO Oxidation by an Atomic Oxygen Beam on $\operatorname{Pt}(111), \operatorname{Ir}(111)$, and $\mathrm{Ru}(001)$. J. Chem. Phys. 1998, $108,3057-3063$.

(23) Ueta, H.; Gleeson, M. A.; Kleyn, A. W. Scattering of Hyperthermal Nitrogen Atoms from the Ag(111) Surface. J. Phys. Chem. A 2009, 113, 15092-15099.

(24) Ueta, H.; Gleeson, M. A.; Kleyn, A. W. The Interaction of Hyperthermal Nitrogen with Ncovered Ag(111). J. Chem. Phys. 2011, 135, 074702.

(25) Quintas-Sánchez, E.; Larrégaray, P.; Crespos, C.; Martin-Gondre, L.; Rubayo-Soneira, J.; J.-C. Rayez, Dynamical Reaction Pathways in Eley-Rideal Recombination of Nitrogen from W(100). J. Chem. Phys. 2012, 137, 064709.

(26) Vanderbilt, D. Soft Self-Consistent Pseudopotentials in a Generalized Eigenvalue Formalism. Phys. Rev. B 1990, 41, 7892-7895.

(27) Perdew, J. P.; Chevary, J. A.; Vosko, S. H.; Jackson, K. A.; Pederson, M. R.; Singh, D. J.; Fiolhais, C. Atoms, Molecules, Solids, and Surfaces - Applications of the Generalized Gradient Approximation for Exchange and Correlation. Phys. Rev. B 1992, 46, 6671-6687.

(28) Busnengo, H. F.; Salin, A.; Dong, W. Representation of the 6D Potential Energy Surface for a Diatomic Molecule Near a Solid Surface. J. Chem. Phys. 2000, 112, 7641-7651.

(29) Theoretical details can be found in the Supplementary Information.

(30) Adelman, S. A.; Doll, J. D. Generalized Langevin Equation for Atom-Solid-Surface Scattering - General Formulation for Classical Scattering off Harmonic Solids. J. Chem. Phys. 1976, $64,2375-2388$. 
(31) Adelman, S. A. Generalized Langevin Theory for Many-Body Problems in Chemical Dynamics - General Formulation and the Equivalent Harmonic Chain Representation. J. Chem. Phys. 1979, 71, 4471-4486.

(32) Tully, J. C. Dynamics of Gas-Surface Interactions - 3D Generalized Langevin Model Applied to fcc and bcc Surfaces. J. Chem. Phys. 1980, 73, 1975-1985.

(33) Busnengo, H. F.; Di Césare, M. A.; Dong, W.; Salin, A. Surface Temperature Effects in Dynamic Trapping Mediated Adsorption of Light Molecules on Metal Surfaces: H-2 on Pd(111) and Pd(110). Phys. Rev. B 2005, 72, 125411.

(34) Martin-Gondre, L.; Alducin, M.; Bocan, G. A.; Díez Muiño, R.; Juaristi, J. I. Competition Between Electron and Phonon Excitations in the Scattering of Nitrogen Atoms and Molecules off Tungsten and Silver Metal Surfaces. Phys. Rev. Lett. 2012, 108, 096101.

(35) Martin-Gondre, L.; Bocan, G. A.; Blanco-Rey, M.; Alducin, M.; Juaristi, J. I.; Díez Muiño, R. Scattering of Nitrogen Atoms off Ag(111) Surfaces: A Theoretical Study. J. Phys. Chem. C 2013, 117, 9779-9790.

(36) Blanco-Rey, M.; Martin-Gondre, L.; Díez Muiño, R.; Alducin, M.; Juaristi, J. I. Dynamics of Nitrogen Scattering off N-Covered Ag(111). J. Phys. Chem. C 2012, 116, 21903-21912.

(37) Ponjée, M. W. G.; Flipse, F. J.; Denier van der Gon, A. W.; Brongersma, H. H. Experimental Observation of Vibrational Modes on $\operatorname{Ag}(111)$ Along $\overline{\Gamma M}$ and $\overline{\Gamma K}$. Phys. Rev. B 2003, 67, 174301.

(38) Martin-Gondre, L.; Bocan, G. A.; Alducin, M.; Juaristi, J. I.; Díez Muiño, R. Energy Dissipation Channels in the Adsorption of $\mathrm{N}$ on $\mathrm{Ag}(111)$. Comp. Theo. Chem. 2012, 990, 126-131.

(39) Tully, J. C. Dynamics of Gas-Surface Interactions - Reaction of Atomic Oxygen with Adsorbed Carbon on Platinum. J. Chem. Phys. 1980, 73, 6333-6342. 
(40) Quintas-Sánchez, E.; Crespos, C.; Larrégaray, P.; J.-C. Rayez,; Martin-Gondre, L.; RubayoSoneira, J. Surface Temperature Effects on the Dynamics of $\mathrm{N}_{2}$ Eley-Rideal Recombination on W(100). J. Chem. Phys. 2013, 138, 024706.

(41) Lahaye, R. J. W. E.; Kang, H. Mechanism for the Efficient Abstraction of an Adsorbate by $\mathrm{Cs}^{+}$Scattering at Hyperthermal Energies. Phys. Rev. B 2003, 67, 033401.

(42) Lahaye, R. J. W. E.; Kang, H. Reactive Ion Surface Scattering as an Eley-Rideal Process: a Molecular Dynamics Study into the Abstraction Reaction Mechanism by Low Energy Cs ${ }^{+}$ from Pt(111). ChemPhysChem 2004, 5, 697.

(43) J.-Y. Kim,; Lee, J. Spatial and Kinetic Separation of Eley-Rideal plus Primary Hot Atom and Secondary Hot Atom Mechanisms in H Atom Abstraction of Adsorbed D Atoms on Pt(111). Phys. Rev. Lett. 1999, 82, 1325-1328.

(44) J.-Y. Kim,; Lee, J. Kinetics, Mechanism, and Dynamics of the Gas-Phase H(D) Atom Reaction with Adsorbed D(H) Atom on Pt(111). J. Chem. Phys. 2000, 113, 2856-2865. 


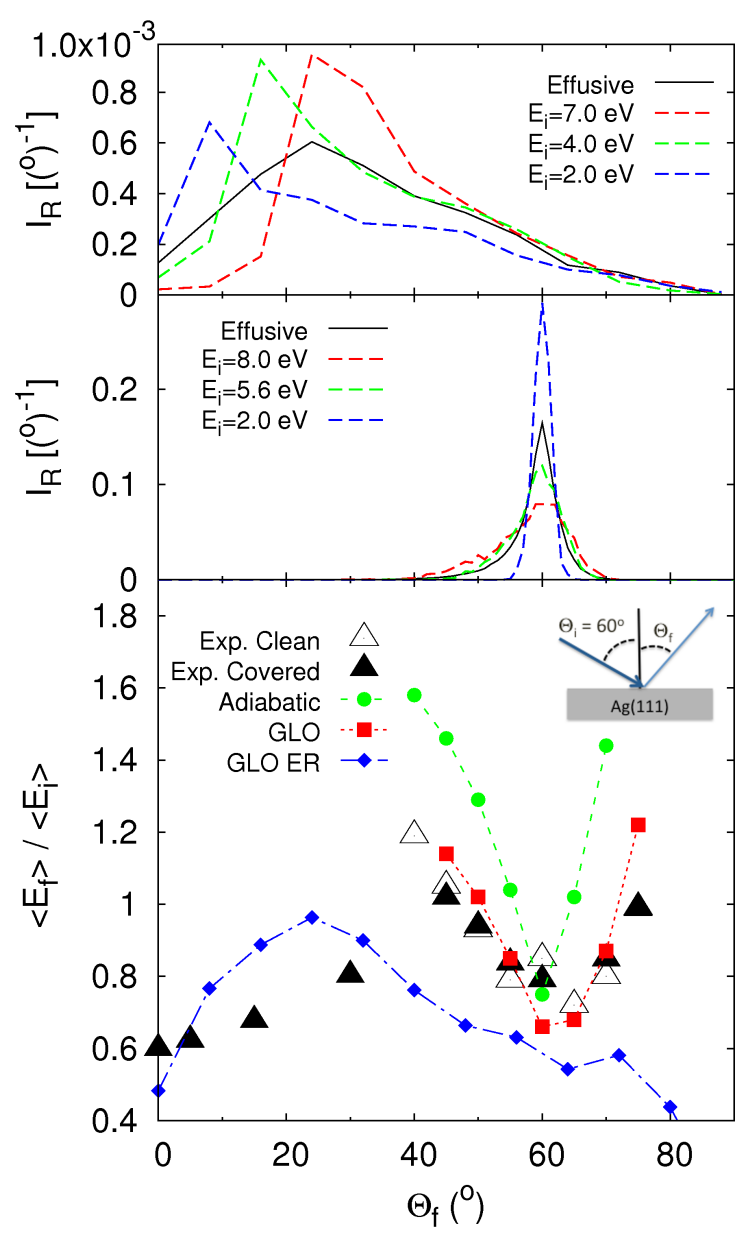

Figure 1: Top panel: angular distribution of $\mathrm{N}_{2}$ molecules formed by ER upon atomic $\mathrm{N}$ scattering on $\mathrm{N}$-covered $\operatorname{Ag}(111)$, which leave the surface at in-plane directions in GLO simulations. Middle panel: angular distribution of $\mathrm{N}_{2}$ reflected in-plane off clean $\mathrm{Ag}(111)$ in an adiabatic frozen surface simulation. In these two panels, the solid (dashed) line corresponds to an effusive (monoenergetic) beam. Bottom panel: angular distribution of the final-to-initial energy ratio of in-plane detected $\mathrm{N}_{2}$. Large open and filled triangles correspond to the experimental results of Ref. ${ }^{24}$ on clean and $\mathrm{N}$-covered $\mathrm{Ag}(111)$. Small symbols are theoretical results from MD using effusive incident beams. Small circles and squares are obtained from adiabatic frozen surface and GLO simulations, respectively, of $\mathrm{N}_{2}$ reflected off clean $\mathrm{Ag}(111)$, and small diamonds correspond to $\mathrm{N}_{2}$ formed by ER of $\mathrm{N}$ scattering on $\mathrm{N}$-covered $\mathrm{Ag}(111)$. The inset shows the in-plane scattering geometry. 


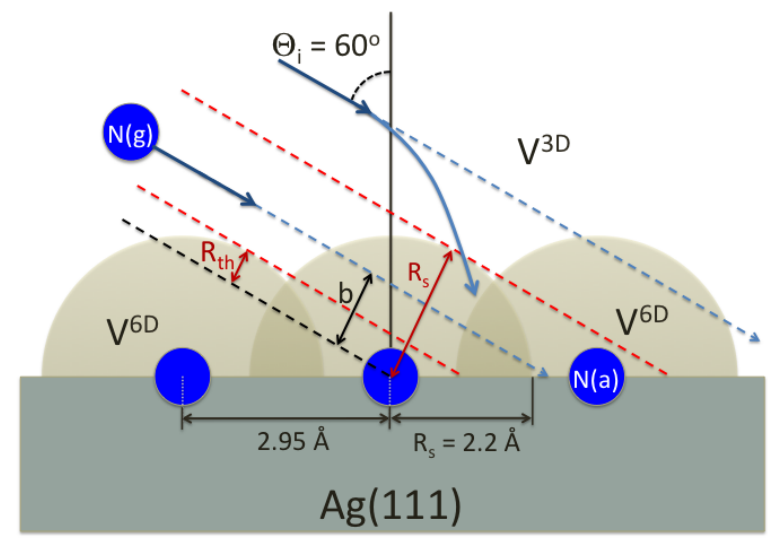

Figure 2: Schematic view of the incidence geometry used to simulate the ER events. The spheres with radius $R_{S}$ denote the range around each surface adsorbate where the 6D PES is to be used. The impact parameter $b$ is defined as the minimum distance between the $\mathrm{N}(\mathrm{g})$ incident direction at time $t=0$ and the target $\mathrm{N}(\mathrm{a})$. The curved trajectory describes a case of projectile steering in the $V^{3 D}$ region.

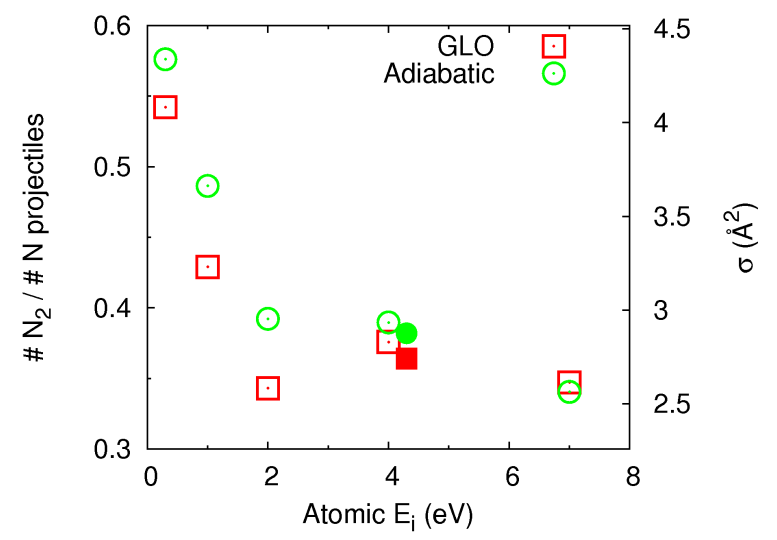

Figure 3: The open symbols denote the efficiency of the ER process as a function of the incident $\mathrm{N}(\mathrm{g})$ kinetic energy $E_{i}$ in simulations of atomic $\mathrm{N}$ scattering on $\mathrm{N}$-covered $\mathrm{Ag}(111)$. Filled symbols account for the efficiencies obtained when an effusive atomic beam of $\left\langle E_{i}\right\rangle=4.3 \mathrm{eV}$ is used. The ER cross-section, $\sigma$, can be formulated as the efficiency times the area per adsorbed atom. 


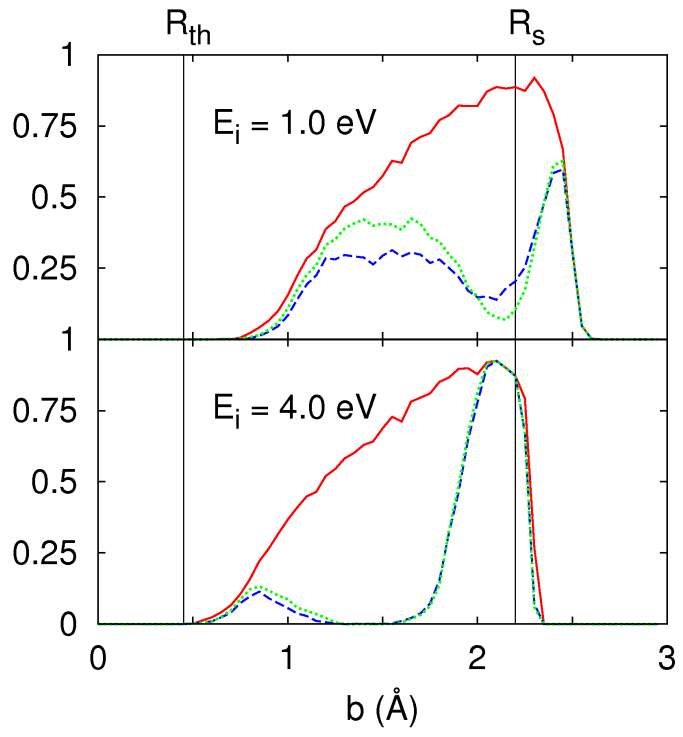

Figure 4: Initial impact parameter distributions of all the incident $\mathrm{N}(\mathrm{g})$ atoms, $P_{0}$ (solid line), and the ones that result in ER reactions of atomic $\mathrm{N}$ on $\mathrm{N}$-covered $\operatorname{Ag}(111), P_{r}$, obtained from GLO (dashed line) and adiabatic frozen surface calculations (dotted line). The vertical lines indicate the geometrical threshold values (see text) for the PES boundary choice made in the present work, $R_{S}=2.2 \AA$. 


\title{
Supporting information for: "Efficient $\mathbf{N}_{2}$ Formation
}

\section{on $\mathrm{Ag}(111)$ by Eley-Rideal Recombination of Hyperthermal Atoms"}

\author{
María Blanco-Rey, ${ }^{*}, \dagger$ Estibaliz Díaz, ${ }^{\ddagger}$ Gisela A. Bocan, ${ }^{\mathbb{I}}$ Ricardo Díez Muiño,, \\ Maite Alducin, ${ }^{\S} \ddagger$ and J. Iñaki Juaristi $\AA^{\S} \dagger, \$$
}

Departamento de Física de Materiales, Facultad de Químicas UPV/EHU, Apartado 1072, 20018 Donostia-San Sebastián, Spain, Donostia International Physics Center (DIPC), Paseo Manuel de Lardizabal 4, 20018 Donostia-San Sebastián, Spain, CONICET and Centro Atómico Bariloche (CNEA), Av. Bustillo 9500, 8400 S.C. de Bariloche, Argentina, and Centro de Física de Materiales CFM/MPC (CSIC-UPV/EHU), Paseo Manuel de Lardizabal 5, 20018 Donostia-San Sebastián, Spain

E-mail: maria.blanco@ehu.es

\footnotetext{
${ }^{*}$ To whom correspondence should be addressed

† Departamento de Física de Materiales, Facultad de Químicas UPV/EHU, Apartado 1072, 20018 Donostia-San Sebastián, Spain

¥ Donostia International Physics Center (DIPC), Paseo Manuel de Lardizabal 4, 20018 Donostia-San Sebastián, Spain

Il CONICET and Centro Atómico Bariloche (CNEA), Av. Bustillo 9500, 8400 S.C. de Bariloche, Argentina

$\S$ Centro de Física de Materiales CFM/MPC (CSIC-UPV/EHU), Paseo Manuel de Lardizabal 5, 20018 DonostiaSan Sebastián, Spain
} 


\section{Construction of the 6D PES}

The interaction energy of the $\mathrm{N}_{2}$ molecule with the $\operatorname{Ag}(111)$ surface is described with a full adiabatic six-dimensional (6D) potential energy surface (PES) that depends on the center of mass position $\mathbf{R}_{C M}=\left(X_{C M}, Y_{C M}, Z_{C M}\right)$ and the internal coordinates $(r, \theta, \varphi)$ of the molecule. The PES is constructed from the interpolation of 6578 ab-initio energies corresponding to 11 values for $r$, 23 for $Z_{C M}$ and 26 for different configurations of $\left(X_{C M}, Y_{C M}, \theta, \varphi\right)$.

All ab-initio data are obtained using the DFT "Vienna Ab initio Simulation Program" (VASP) code ${ }^{1}$ that operates with a plane-wave basis set. The exchange and correlation energy is calculated with the generalized gradient approximation (GGA) and the Perdew-Wang energy functional (PW91) . ${ }^{2}$ The electron-core interaction is described with ultra-soft pseudopotentials . ${ }^{3}$ Preliminary calculations have been performed to ensure that all $a b$ initio energies are calculated to a prescribed accuracy. Thus, the energy cutoff in the plane-wave expansion is $453 \mathrm{eV}$; the fractional occupancies are determined through the broadening approach of Methfessel and Paxton ${ }^{4}$ with $N=1$ and $\sigma=0.2$; and, given the surface's hexagonal geometry, the Brillouin-zone integration is performed with a $5 \times 5 \times 1$ Gamma Centered Grid of special k points. ${ }^{5}$

The theoretical lattice constant obtained from a bulk calculation is $a=4.17 \AA$. This leads to an interlayer spacing of $d=2.408 \AA$. The $\mathrm{Ag}(111)$ surface is modeled by a 5-layer slab with a supercell vector along the normal to the surface ( $O Z$ axis) of $10 d$. Under these conditions and keeping the third layer fixed, the interlayer distance is relaxed to get the surface equilibrium geometry. These relaxation corrections are however found to be almost negligible (around $0.1 \%$ ) for both the first and second interlayer distances. Once obtained, the relaxed geometry of the slab is kept frozen for the calculations that follow. The ab-initio energies for the $\mathrm{N}_{2} / \mathrm{Ag}(111)$ system are obtained using a $(2 \times 2)$ surface structure $(0.25$ coverage $)$. The energies are referred to that of the $\mathrm{N}_{2}$ molecule located parallel to the surface and midway between the two slabs, which is the simulated asymptotic region.

Once the 6578 point energy grid is obtained, the PES is built by interpolating the ab-initio data. The Corrugation Reducing Procedure ${ }^{6}$ is used to obtain an auxiliary, smooth, and easy 
to interpolate function $F(X, Y, Z)$, which is spline interpolated along the $Z_{C M}$ and $r$ coordinates and 2D Fourier interpolated within the $X_{C M} Y_{C M}$ plane and the angular internal coordinates. The interpolated value of $F(X 0, Y 0, Z 0)$ can easily be converted back to an interpolated system energy $E(X 0, Y 0, Z 0)$. Note that the $2 \mathrm{D}$ Fourier expansion is here chosen over a $2 \mathrm{D}$ Periodic Spline in order to profit from the fact that it analytically respects the surface hexagonal symmetry. The accuracy of the constructed 6D PES has been checked by comparing its output to a set of ab-initio off-grid values. The errors are found to be of just a few tens of meV.

\section{Derivative continuity checks at the boundary between the 3D}

\section{and the 6D PES}

Eley-Rideal (ER) recombination of gas-N, N(g), and adsorbed-N on $\mathrm{Ag}(111)$ at saturation coverage, N(a), has been simulated making use of two PESs calculated ab-initio. In each MD trajectory, the $\mathrm{N}(\mathrm{g})$ projectile departs from an initial height $z_{i}=6 \AA$ over the $\operatorname{Ag}(111)$ topmost layer and $\left(x_{i}, y_{i}\right)$ coordinates generated by a conventional Monte Carlo (MC) sampling. Its incidence direction is defined by a fixed polar angle $\Theta_{i}=60^{\circ}$, measured with respect to the surface normal, and a random azimuthal angle $\Phi_{i}$ generated by MC sampling, too. The hyperthermal kinetic energies have been analysed in the present work are $E_{i}=0.3,1.0,2.0,4.0,7.0 \mathrm{eV}$, plus an effusive beam of $\mathrm{N}(\mathrm{g})$ gas atoms that matches the experimental atomic beam energy distribution, with average energy $\left\langle E_{i}\right\rangle=4.3 \mathrm{eV}$ and $f w h m=5.4 \mathrm{eV} .^{7,8}$ For each energy, $N_{\text {traj }}=8 \times 10^{5}$ trajectories are simulated that will be used to extract statistical averages. Classical molecular dynamics are performed by integrating the equations of motion with a time interval $h \leq 10^{-2}$ fs.

As explained in the manuscript, the simulation space is divided in two parts:

(i) Previously to the collision, at distances $R>R_{s}$ from the $\mathrm{N}(\mathrm{a})$ species, the $\mathrm{N}(\mathrm{g})$ dynamics are dictated by the 3D PES on the $(1 \times 1) \mathrm{N}$-covered $\operatorname{Ag}(111)$ surface, $V^{3 D}$, which is described in detail in Ref. ${ }^{9}$ At this stage of the MD, surface atoms are kept fixed at their adorption sites. 
(ii) When the projectile approaches the surface and the distance between the $\mathrm{N}(\mathrm{g})$ atom and a $\mathrm{N}$ (a) atom on the surface is $R<R_{s}$, the latter atom is allowed to move, and the interaction between these two atoms evolves under a $6 \mathrm{D}$ PES, $V^{6 D}$, namely that of a $\mathrm{N}_{2}$ molecule interacting with a clean $\operatorname{Ag}(111)$ surface. At this stage, recombination can take place.

Since the the influence of neighbouring adsorbates is neglected at stage (ii) of the MD, we must dismiss from the analysis channels other than $\mathrm{N}_{2}$ formation by an ER process with fast interaction with the surface. For the effusive atomic beam, we find average reaction times smaller than 0.4 ps. Other channels, such as adsorption or trapping, may prelude molecule recombination by hot atoms or Langmuir-Hinshelwood mechanisms at longer reaction times after interaction with several adsorbates.

The methodology above will constitute a reliable model for the ER reaction as long as $\mathrm{N}(\mathrm{g})$ experiences a small discontinuity when crossing the boundary between the two PESs at $R=R_{s}$. After a first stage evolving under $V^{3 D}, \mathrm{~N}(\mathrm{~g})$ arrives at the boundary with position $\mathbf{x}_{s}$ and velocity $\dot{\mathbf{x}}_{s}$. These are the initial conditions for the second part of the simulation with $V^{6 D}$. The amount of energy not conserved because of switching to another PES, $\delta E\left(R_{S}\right)$, can be estimated from the discontinuity in the force experienced by $\mathrm{N}(\mathrm{g})$ at the boundary using:

$$
\delta E\left(R_{S}\right) \approx h \dot{\mathbf{x}}_{s} \cdot\left[\nabla V^{3 D}\left(\mathbf{x}_{s}\right)-\nabla V^{6 D}\left(\mathbf{x}_{s}\right)\right]+\mathscr{O}\left(h^{2}\right)
$$

Fig. Figure 1 shows the distributions of $|\delta E|$ for the studied trajectories, calculated for the largest used time step, $h=0.01 \mathrm{fs}$, and several values of $E_{i}$ and $R_{s}$. In all the cases $|\delta E|$ is of the order of a few meV, much smaller than the incident energies. Distributions are broader for larger $E_{i}$ and for smaller $R_{s}$. The choice made in the present work, $R_{s}=2.2 \AA$, ensures that the energy discontinuities will be $\lesssim 2 \%$ of the $E_{i}$ value with very few exceptions. Typically, the trajectories with large $|\delta E|$ are those that enter the $R<R_{S}$ region at small $z$ values, i.e. they experience a stronger interaction with neighbouring N(a) in region (i) that is abruptly switched off when they enter region (ii). 


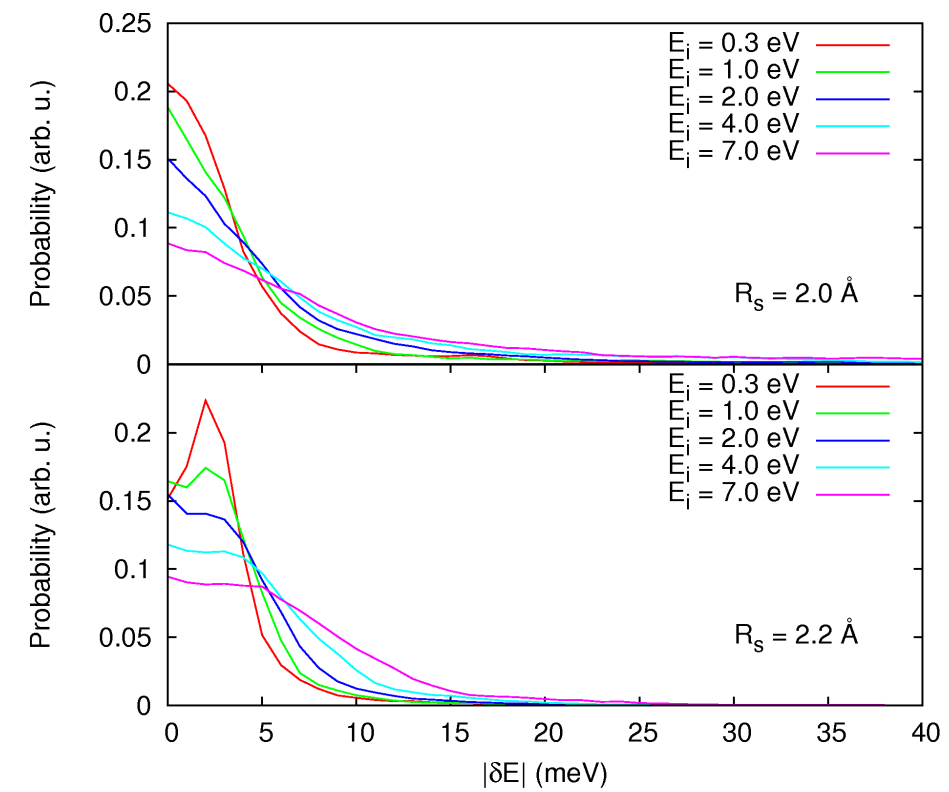

Figure 1: Energy discontinuity distributions at the boundary between the 6D and 3D PES regions for $E_{i}$ values studied in the present work. Each panel accounts for two locations $R_{S}$ of the boundary.

\section{References}

(1) Kresse, G.; Hafner, J. Ab Initio Molecular-Dynamics for Liquid-Metals. Phys. Rev. B 1993, $47,558$.

(2) Perdew, J. P.; Chevary, J. A.; Vosko, S. H.; Jackson, K. A.; Pederson, M. R.; Singh, D. J.; Fiolhais, C. Atoms, Molecules, Solids, and Surfaces - Applications of the Generalized Gradient Approximation for Exchange and Correlation. Phys. Rev. B 1992, 46, 6671-6687.

(3) Vanderbilt, D. Soft Self-Consistent Pseudopotentials in a Generalized Eigenvalue Formalism. Phys. Rev. B 1990, 41, 7892-7895.

(4) Methfessel, M.; Paxton, A. T. High-Precision Sampling for Brillouin-Zone Integrations in Metals. Phys. Rev. B 1989, 40, 3616.

(5) Monkhorst, H. J.; Pack, J. D. Special Points for Brillouin-Zone Integrations. Phys. Rev. B 1976, $13,5188$. 
(6) Busnengo, H. F.; Salin, A.; Dong, W. Representation of the 6D Potential Energy Surface for a Diatomic Molecule Near a Solid Surface. J. Chem. Phys. 2000, 112, 7641-7651.

(7) Ueta, H.; Gleeson, M. A.; Kleyn, A. W. The Interaction of Hyperthermal Nitrogen with Ncovered Ag(111). J. Chem. Phys. 2011, 135, 074702.

(8) Martin-Gondre, L.; Alducin, M.; Bocan, G. A.; Díez Muiño, R.; Juaristi, J. I. Competition Between Electron and Phonon Excitations in the Scattering of Nitrogen Atoms and Molecules off Tungsten and Silver Metal Surfaces. Phys. Rev. Lett. 2012, 108, 096101.

(9) Blanco-Rey, M.; Martin-Gondre, L.; Díez Muiño, R.; Alducin, M.; Juaristi, J. I. Dynamics of Nitrogen Scattering off N-Covered Ag(111). J. Phys. Chem. C 2012, 116, 21903-21912. 